\title{
A three-sector endogenous growth model with combined technological change: the choice between basic innovations and quality improvements
}

Citation for published version (APA):

Verberne, P., Muysken, J., \& van Zon, A. H. (1996). A three-sector endogenous growth model with combined technological change: the choice between basic innovations and quality improvements. MERIT, Maastricht Economic Research Institute on Innovation and Technology. MERIT Research Memoranda No. $011 \mathrm{https}: / /$ doi.org/10.26481/umamer.1996011

Document status and date:

Published: 01/01/1996

DOI:

10.26481/umamer.1996011

Document Version:

Publisher's PDF, also known as Version of record

Please check the document version of this publication:

- A submitted manuscript is the version of the article upon submission and before peer-review. There can be important differences between the submitted version and the official published version of record.

People interested in the research are advised to contact the author for the final version of the publication, or visit the DOI to the publisher's website.

- The final author version and the galley proof are versions of the publication after peer review.

- The final published version features the final layout of the paper including the volume, issue and page numbers.

Link to publication

\footnotetext{
General rights rights.

- You may freely distribute the URL identifying the publication in the public portal. please follow below link for the End User Agreement:

www.umlib.nl/taverne-license

Take down policy

If you believe that this document breaches copyright please contact us at:

repository@maastrichtuniversity.nl

providing details and we will investigate your claim.
}

Copyright and moral rights for the publications made accessible in the public portal are retained by the authors and/or other copyright owners and it is a condition of accessing publications that users recognise and abide by the legal requirements associated with these

- Users may download and print one copy of any publication from the public portal for the purpose of private study or research.

- You may not further distribute the material or use it for any profit-making activity or commercial gain

If the publication is distributed under the terms of Article 25fa of the Dutch Copyright Act, indicated by the "Taverne" license above, 


\section{A Three-Sector Endogenous Growth Model with Combined Technological Change: \\ the choice between basic innovations and quality improvements}

by

P. Verberne

A.H. van Zon

J. Muysken

Maastricht

June 24, 1996 


\section{Technological change, expansion and improvement}

Since Solow (1956), technological change is regarded as one of the main sources of economic growth. Without continuous improvements of the technologies we use and without the discovery of totally new technologies, growth depends on the 'balanced' accumulation of the physical factors of production only. Using neo-classical marginal productivity assumptions, technological change (or labour growth) is needed to compensate for the negative productivity effects of capital accumulation. Although the importance of technological change is widely accepted, the almost casual (exponential) specification of growth models which incorporate the use of this concept, does not match its overriding importance. Especially in models which concern the environment, technological change takes an important place in practice. Most of these models, which are used to evaluate environmental policy, treat technological change exogenously [c.f. Verberne (1995)]. The outcomes of these models depend widely on the rate of technological change chosen and the number of backstop technologies available at a certain point in time. Taking an exogenous rate of exponential technological change, and keeping it constant over a relatively long period of time, might give too optimistic a view of the future. In addition, this assumption neglects the fact that new technologies depend on R\&D expenditures, investment decisions and economic policy. From these practical observations we concluded that, especially in light of the outcomes of these environmental models and their far reaching conclusions, technological change should be determined endogenously rather than exogenously.

The concern for endogenous technological change has resulted in the emergence of the so called 'new growth theory'. This endogenous growth literature provides us with better insights in the causes and effects of technological change as a determinant of economic growth. Basically we can distinguish two different types of technological change: an increase in the number of technologies on the one hand [c.f. Romer (1990), Grossman \& Helpman (1991)] and a quality improvement of the existing technologies [c.f. Aghion \& Howitt (1992), Grossman \& Helpman (1991)] on the other. The first type of technological change can be compared, at least in terms of its effects, with product innovation or embodied technological change. The second type of technological change, improving the quality of already existing technologies, with process innovation or disembodied technological change. It is common practice to use these two types of technological change separately [c.f. Barro \& Sala-i-Martin (1995), Grossman \& Helpman (1991)], although the combination of both new technologies and quality improvements is 
suggested by Helpman \& Trajtenberg (1994) ${ }^{1}$. The goal of this paper, however, is to incorporate both notions of technological change in one encompassing technology framework which is as close as possible to the 'standard' optimal control framework used by Lucas (1988) and Romer (1990), for instance. This will result in a model in which both the 'average' type of technological change will be endogenous, and the 'average' rate of technological change. The combination of these two types of technological change is important, considering the possibility that the emergence of new technologies defines potential expansion/growth paths, which the economy can move along. The rate of growth along this path is set by the characteristics of the path itself (exponential versus asymptotic growth paths) and by the resources spent on revealing the potentials of the path through applied R\&D. However, rather than going into these particular issues here, we focus on the outline of the general optimal control framework and the general problem of choosing between basic R\&D on the one hand, which in our interpretation expands the number of technologies available, and applied R\&D on the other, which improves the quality of already known technologies in an incremental way. This particular framework may provide a better insight in the role of technological change in macroeconomic models concerning the environment. We will not, however, in this stage, incorporate this exercise into an environmental model. But intuitively it is clear that quality improvements of already existing technologies, i.e. increasing the efficiency of energy generating or energy using technologies, might in some cases have to give way to investments in totally new technologies, especially in case of asymptotic potential growth paths.

In this paper we will focus on a situation of steady state growth in a social optimum. In the first two sections, $R \& D$ growth models based on either an increase in the number of technologies or the improvement of already existing technologies will be summarized. In section three both notions of technological change will be compared, using the concept of the marginal growth productivity of human capital. In section four a first attempt is made to incorporate both notions of technological change into one model using standard 'constant returns' assumptions with respect to $R \& D$ in generating growth, introducing a two-step approach. In the case of constant returns technology functions, the solution of this model leads to corner solutions

\footnotetext{
${ }^{1}$ In their paper, Helpman and Trajtenberg distinguish general purpose technologies (GPTs), such as the invention of the steam engine and electricity, and complementary innovations in which the GPTs are used. Their approach differs from the approach taken in this paper because we do not focus on the complementary 'offspring' of these new technologies but on the improvement of specific technologies.
} 
rather than interior solutions, in which case again only one of the two types of technological change is economically relevant. The next sections show that by dropping the assumption of constant returns in the technology equations a steady state solution with a non-trivial technology mix can be obtained. The paper ends with a summary and some suggestions regarding the future use and expansion of the framework.

\section{Expanding the number of technologies}

Technological change by means of an increase in the number of available technologies will be discussed using a simplified version of the Romer (1990) model. In this simplified version of the Romer model human capital $(H)$ and physical capital $(K)$, together with the level of existing technology $(A)$, are the only factors of production. This makes the model easier to handle without changing the essential features and outcomes of the model. As in the Romer (1990) model, two types of knowledge are distinguished. The first type of knowledge consists of human capital which can be used to generate blueprints. The total number of blueprints reflects the total stock of directly productive knowledge available to the economy. Human capital is a rival good because its use by one firm precludes its use by another firm. Technology, on the other hand, is non-rival because its use by the one firm does not limit its use to another firm. Human capital can be used both for the production of the final output $(Y)$ and the generation of new technologies. In the R\&D sector, new technologies are generated by using human capital and the stock of knowledge, which is proportional to the number of blueprints. Therefore the technology generation equation can be written as follows,

$$
\frac{d A}{d t}=\delta v H A
$$

where $v$ is the fraction of the total stock of human capital devoted to the $\mathrm{R} \& \mathrm{D}$ sector and $\delta$ is a productivity parameter. Note that (1.1) implies that for a constant value of $v H$, growth itself is constant. The amount of human capital devoted to the R\&D sector and the amount of human capital devoted to the final goods sector equals the total amount of human capital. From (1.1) it is also clear that human capital is a scale variable and that an exogenous increase in the total stock of human capital implies a higher rate of growth of the level of knowledge. If we would scale human capital to one from equation (1.1) onwards, we would in essence have the same model as in Lucas (1988), in which the growth of human capital has the same function as the knowledge in the Romer (1990) model. In the Lucas (1988) model labour is the scale variable and is scaled to one. Note that the research sector is human-capitalintensive and technology-intensive. Physical capital $(K)$ does not enter the 
technology equation; it is used in the production of final goods only,

$$
Y=[(1-v) H]^{\alpha} \cdot \sum_{i=1}^{A}\left(x_{i}\right)^{1-\alpha}
$$

where $x_{i}$ represents the amount of capital of type $i$. This Cobb-Douglas type production function is based on an Ethier (1982) production function were output is an additively separable function of different types of capital goods each of them built in accordance with a different blueprint $x_{i}$. Since all $x_{i}$ enter the production function symmetrically ${ }^{2}$ we can write (1.2) directly as,

$$
Y=[(1-v) H]^{\alpha} \cdot K^{1-\alpha} \cdot A^{\alpha}
$$

As in the Romer (1990) model we will assume that the growth rate of human capital is zero ${ }^{3}$. An increase in the share of human capital devoted to the generation of new technologies will increase the growth rate of $A$ and therefore final output indirectly. But it will also decrease, at least in the short run, final output because of the decrease in human capital available for final production.

In order to look at the long run effects of changes in the share of human capital devoted to either final production or the generation of new technologies and the steady state growth rates, we will derive the necessary conditions for a social optimum from the maximization of the present value of an infinite stream of consumer utility $(U)$,

$$
U=\int_{0}^{\infty} e^{-\rho t} \frac{C^{1-\theta}-1}{1-\theta} d t
$$

where $\mathrm{C}$ is consumption. The intertemporal substitution parameter, $\theta$, lies between zero and one and the rate of time preference, $\rho$, is always positive. This utility function is called the constant intertemporal elasticity of substitution (CIES) utility function, because the elasticity of substitution between units of consumption at different points in time is constant and equal to $1 / \theta$. A higher value of $\theta$ implies that consumers are less willing to substitute consumption possibilities at different points in time [Barro \& Salai-Martin (1995)], i.e. consumers are less willing to trade current consumption for future consumption. The socially optimal rate of growth can be obtained

\footnotetext{
${ }^{2}$ The aggregation over the individual blueprints, in order to obtain effective capital, is explained in more detail in Appendix A.

${ }^{3}$ Assuming zero population growth and assuming that the accumulation of human capital is the result of schooling and on the job training which is lost at the end of a lifetime, the net cumulative effect is zero growth in human capital.
} 
by means of intertemporal maximization of the present value of total utility $(U)$ over an infinite horizon, subject to the technology generation equation (1.1) and the economy's budget constraint,

$$
\frac{d K}{d t}=Y-C
$$

This results in ${ }^{4}$,

$$
v=\frac{\delta H-\rho}{\theta \delta H}
$$

where $v$ represents the fraction of human capital allocated to the generation of new technologies. From this it follows that $\delta H$ is the productivity parameter of $v$. Note that again the scale effect of human capital is present. An exogenous increase in the stock of human capital would increase the share of this stock allocated to the generation of new technologies. Since $v$ has to be non-negative and at most equal to one, it has to be the case that $\rho \leq \delta H \leq \frac{\rho}{1-\theta}$. Note that $v$ depends positively on the productivity parameter $\delta$ and human capital. A higher productivity of human capital in the technology generating sector induces a higher share of human capital to be allocated to this sector. The positive relation between $v$ and the stock of human capital implies that there is some sort of scale effect. An exogenous increase in the stock of human capital increases the share of this stock allocated to the generation of new technologies. In models in which the stock of human capital grows [c.f. Lucas (1988)], this effect is absent. This is obvious since an increase in human capital would in that case imply an ever increasing share of human capital allocated to the generation of new technologies. The share depends, furthermore, negatively on the rate of time preference and the intertemporal substitution parameter $\theta$. A higher rate of time preference implies that a lower fraction of human capital is devoted to the production of new technologies. In other words, present production (and consumption) is preferred over future consumption possibilities.

Since the steady state growth rate of the various quantities equals the common growth rate, we know from equation (1.1) that $g=v \delta H$, so,

$$
g=\frac{\delta H-\rho}{\theta}
$$

This shows that a shift from future to present consumption, caused by an

\footnotetext{
${ }^{4}$ See Appendix A for a derivation of the results.
} 
increase in the rate of time preference, decreases the steady state growth rate caused by a reduction of the amount of human capital allocated to increasing future consumption potentials through research, thus stressing the importance of $R \& D$ in generating future consumption possibilities. Note that, because of the fact that human capital is a scale variable, a higher stock of human capital implies a higher growth rate.

\section{Improving already existing technologies}

The other way in which technological change can be modelled is to treat technological change as the increase in quality of a fixed number of already existing technologies. This notion can be found in Aghion \& Howitt (1992) and Grossman \& Helpman (1991). Since we want to incorporate both types of technological change into one model, they are modelled in as similar a way as possible. In our set-up, the source of quality increases is assumed to be the use of human capital. As in the previous section we will divide the total available amount of human capital between final production and the generation of technological change.

We can rewrite the production function from the previous section somewhat, so as to be more in line with the production function used by Barro \& Sala-iMartin (1995),

$$
Y=[(1-v) H]^{\alpha} \cdot \sum_{i=1}^{A}\left(q x_{i}\right)^{1-\alpha}
$$

In this production function the number of technologies, $A$, is fixed. Note that an increase in the quality, $q$, increases the total efficiency of all designs (or capital goods), $x_{i}$, and increases therefore total output, $Y^{5}$ As in the previous section we can rewrite this production function and replace all the separate capital goods (or designs), $x_{i}$, by an expression in $q, A$ and $K$,

$$
Y=[(1-v) H]^{\alpha} \cdot(q K)^{1-\alpha} \cdot A^{\alpha}
$$

The engine of growth is no longer the increase in the number of technologies, as expressed in equation (1.1), but the increase in quality of existing technologies. The relevant technology generation equation is now,

$$
\frac{d q}{d t}=\delta v H q
$$

As in the previous section, we assume that only human capital is needed to

\footnotetext{
${ }^{5}$ As in Appendix A, the capital aggregate can be seen as a linear homogeneous CES aggregate of effective capital. The efficiency is in this case positively affected by an increase in quality.
} 
improve the quality of already existing technologies. Human capital is still a scale variable in the sense that an exogenous increase in the stock of human capital increases the growth rate of quality improvements.

Intertemporal utility maximization from the point of view of the social planner, results in 6 ,

$$
v=\frac{\delta H \frac{1-\alpha}{\alpha}-\rho}{\theta \delta H \frac{1-\alpha}{\alpha}}
$$

Note the complete similarity between (2.4) and (1.7). Again a higher rate of time preference implies a lower share of human capital devoted to generating technological change. The difference between both equations is the term $\frac{1-\alpha}{\alpha}$. This term will be interpreted in more detail in the next section.

Since the steady state growth rate of the various variables equals the common growth rate, we know that,

$$
g=\hat{K}=\hat{Y}=\hat{C}=\frac{1-\alpha}{\alpha} \hat{q}=\frac{1-\alpha}{\alpha} \delta v H
$$

Substitution of equation (2.4) into this equation results in,

$$
g=\frac{\delta H \frac{1-\alpha}{\alpha}-\rho}{\theta}
$$

This shows that a shift from future to present consumption, caused by an increase in the discount rate, decreases the steady state growth rate. Again the close relation between the growth rate in this section and the previous section is obvious.

\section{The Marginal Growth Productivity of Human Capital}

As noticed in the previous sections, both notions of technological change are almost identical, at least in terms of their growth rates and the amount of human capital allocated to the generation of technological change. The

\footnotetext{
${ }^{6}$ See Appendix B for a derivation of the results presented here.
} 
interpretation of both notions will become even more clear if we rewrite equations (2.4) and (2.6) to,

$$
v=\frac{\delta^{\prime} H-\rho}{\theta \delta^{\prime} H} \quad g=\frac{\delta^{\prime} H-\rho}{\theta}
$$

where $\delta^{\prime}=\delta\left(\frac{1-\alpha}{\alpha}\right)$. Note that $\delta$ and $\delta^{\prime}$ represent the change in the steady state values of growth due to allocating an additional unit of human capital to research: $\delta$ and $\delta^{\prime}$ are therefore equal to the marginal growth productivities of human capital, or MGP for short.

Interpreting both cases in terms of the marginal growth productivity of human capital we can simply identify the similarity between the two cases. It is exactly this marginal growth productivity that determines the growth possibilities of both systems. In the representation of the Romer (1990) model the steady state growth rates of $A, K$, and $Y$ were equal. This means that, since

$$
\hat{Y}=\alpha \hat{A}+(1-\alpha) \hat{K}
$$

the marginal growth productivity of human capital equals,

$$
M G P_{A}=\frac{\partial \hat{Y}}{\partial(v H)}=\frac{\partial \hat{A}}{\partial(v H)}=\delta
$$

Note that $v H$ represents here the amount of human capital allocated to the production of new technologies.

In our representation of the framework in which technological change is generated by improving existing technologies, it follows that,

$$
\hat{Y}=(1-\alpha) \hat{q}+(1-\alpha) \hat{K}
$$

Hence, the marginal growth productivity of human capital is,

$$
M G P_{q}=\frac{\partial \hat{Y}}{\partial(v H)}=\left(\frac{1-\alpha}{\alpha}\right) \frac{\partial \hat{q}}{\partial(v H)}=\left(\frac{1-\alpha}{\alpha}\right) \delta=\delta^{\prime}
$$

Note that $v H$ represents here the amount of human capital allocated to the generation of better quality.

Thus, reformulating the results of both notions of technological change in terms of their marginal growth productivity, we can show the similarity between both notions. The factor $\frac{1-\alpha}{\alpha}$ appears due to the different way 
quality improvement and the amount of new technologies influence the production process.

\section{A two-step model of combined technological change}

If we want to incorporate both notions of technological change into one model, the simplest solution would be to just combine the two notions. This would mean that next to an increase in the number of technologies it would also be possible to increase the quality of already existing technologies. This would require two technology equations, one explaining the growth of the number of technologies and the other explaining the growth of the quality of those technologies that already exist. However, the resulting three-sector model can not that easily be solved using the ordinary optimization framework. It can be shown that if we solve the combined technology model using the Hamiltonian approach, the result will be an economically difficult to interpret corner solution (see Appendix C). An intuitively clearer way to solve the three-sector model of combined technological change is to describe it in two steps? ${ }^{7}$. This essentially reduces the three-sector problem of the intertemporal optimization of consumer utility to a two-sector problem. The framework to be developed will make the solution of the model easier and the results better to interpret.

In this two-step approach we will introduce an extra equation which describes the combined technology input, $T$. One can link this combined technology input, which is simply the combination of $A$ and $q$ as found in the original production function, to the total factor productivity (TFP) in the production function,

$$
T F P=\left[A \cdot q^{\frac{1-\alpha}{\alpha}}\right]^{\alpha} \triangleq T^{\alpha} \Leftrightarrow T \hat{F P}=\alpha\left[\hat{A}+\left(\frac{1-\alpha}{\alpha}\right) \hat{q}\right]
$$

Using the combined technology input $T$, rather than the TFP itself, it follows that the production function can be written as,

$$
Y=[(1-z) H]^{\alpha} \cdot K^{1-\alpha} T^{\alpha}
$$

The fractions attached to the allocation of human capital are now redefined in order to clarify the two-step approach we take here. The technology generation equations are changed to,

\footnotetext{
${ }^{7}$ It can be shown, at least for the technological progress functions we have specified, that the two-step approach and the standard approach generate the same results (the formal proof can be found in Appendix D). However, the two-step approach has the advantage over the standard approach of being relatively easy to interpret.
} 


$$
\begin{aligned}
& \frac{d A}{d t}=\delta_{A}(z-u) H A \\
& \frac{d q}{d t}=\delta_{q} u H q
\end{aligned}
$$

Note that $u H$ is now the amount of human capital allocated to quality improvements and $(z-u) H$ is the amount of human capital allocated to the generation of new technologies. The redefinition of the human capital fractions in the context of the two-step approach can best be explained using Figure 1.

The total amount of human capital is allocated in two steps. The first step divides human capital between final production on the one hand and the generation of TFP, through 'combined' technological change $T$, on the other. The fraction $1-z$ is available for final production, while the fraction $z$ of the total stock of human capital will be used in the generation of TFP growth. In the second step the fraction $u$ is allocated to the improvement of quality and the remaining fraction $z-u$ is used to generate totally new technologies ${ }^{8}$.

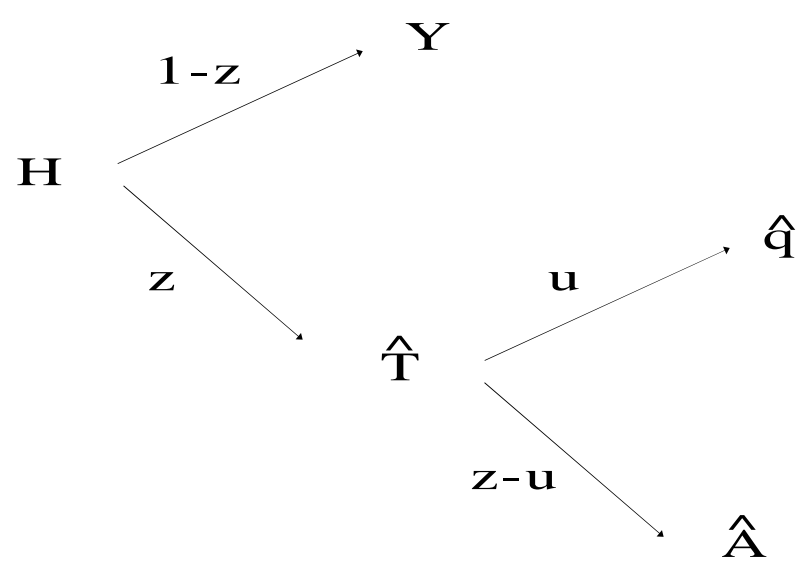

Figure 1 The Two-Step-Approach

Using the two technology generation equations in (4.3), we can derive a combined technology contour (CTC). The CTC is comparable in nature to the invention possibility frontier introduced by Kennedy (1964). It represents all the efficient combinations of growth rates of $A$ and $q$ which can be achieved for a given amount of human capital $(z H)$ allocated to the generation of TFP. The CTC can be obtained directly by solving the $d q / d t$ equation for $u$ and

\footnotetext{
${ }^{8}$ Note however, that it may very well be possible that $z$ itself depends on the way in which human capital can be distributed over $u$ and $z-u$, if the growth rate of $T$ itself depends on that distribution.
} 
substituting this result in the $d A / d t$ equation. So, the CTC defines the trade off between $\hat{q}$ and $\hat{A}$,

$$
\hat{A}=\delta_{A}\left(z-\left(\frac{\hat{q}}{\delta_{q} H}\right)\right) H
$$

The partial derivative of this equation with respect to $\hat{q}$ is equal to $-\delta_{A} / \delta_{q}$. Hence the CTC is downward sloping. Moreover, it is linear in $\hat{q}$ and $\hat{A}$.

Equation (4.1) provides the 'iso-TFP-growth-profile' (ITP) which also gives a relation between $\hat{q}$ and $\hat{A}$. In other words, this ITP depicts the combinations of $\hat{q}$ and $\hat{A}$ in which the combined technology impact on $\hat{T}$ and therefore on TFP-growth is the same. Since intertemporal utility can only be maximized when scarce resources are allocated in an efficient manner, this implies that a given value of $z$ should generate the highest rate of growth of $T$, hence $\hat{Y}$, hence $\hat{C}$, since otherwise the same rate of growth could be realized while reallocating part of $z$ back again to current output production, thus increasing the value of intertemporal utility. Hence, assuming that there is indeed a steady state where, as before, $\hat{q}$ and $\hat{A}$ are constant for constant $H$, it follows that the intertemporal utility is maximized only when a given value of $z$ is distributed over $\hat{q}$ and $\hat{A}$ such that the combined impact of $\hat{q}$ and $\hat{A}$ on $\hat{Y}$ and therefore on $C$ and $\hat{C}$, is also as high as possible. But in the steady state $T$ cannot be maximized without maximizing $\hat{T}$ at the same time. Hence, the problem of distributing $z H$ over $\hat{q}$ and $\hat{A}$ is reduced to the familiar framework of shifting the ITP line outward until it has just one point in common with the linear $\mathrm{CTC}^{9}$. Maximizing the total factor productivity growth therefore implies choosing the highest possible ITP by choosing an optimal combination of $\hat{q}$ and $\hat{A}$. For a concave CTC this requires the slopes of the CTC and the ITP to be the same. For a linear CTC, this may be too strong a requirement, resulting in complete specialization as depicted in Figure 2.

The iso-TFP-growth-profile is represented by the line IIa or IIb, depending on the value of $\alpha$, since it has a slope $-\alpha /(1-\alpha)$. The CTC is represented by line I

\footnotetext{
${ }^{9}$ Note the complete similarity with the Ricardian Trade model. Note too that the same complete specialization conclusions apply here.
} 
and has a slope $-\delta_{q} \delta_{A}$. Whether there will be complete specialization in the production of new technologies or in the quality improvement of existing technologies depends on the slope of the ITP compared to the slope of the CTC.

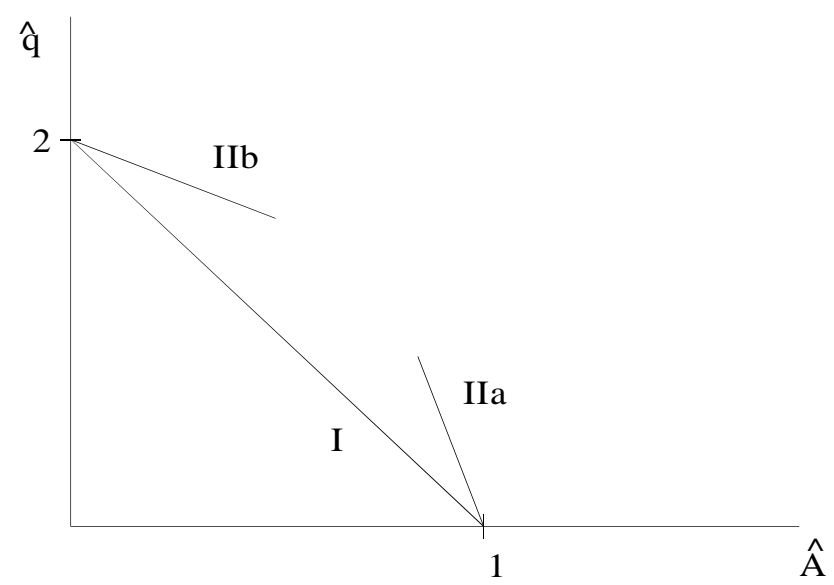

Figure 2 Choice of Technology Types

If the slope of the ITP is smaller (larger) than the slope of the CTC, i.e. line IIb (line IIa), maximizing TFP growth implies complete specialization in the quality improvement of existing technologies (generation of new technologies).

In terms of the MGP interpretation of the human capital allocation process in the previous section, an optimal allocation implies that the MGPs of both technology generating sectors have to be equal. Thus $\delta_{\mathrm{A}}$ has to be equal to $\delta_{q}\left(\frac{1-\alpha}{\alpha}\right)$. Note that this condition is similar to the condition that the slopes of the ITP and the CTC have to be equal.

The analysis so far has only given a theoretical explanation for technological change in case of generating new technologies or improving existing technologies. The two-step approach shows why, in case of linear technology equations, only one of the two notions of technological change is relevant in practice. Obviously, if we want to incorporate both notions of technological change into one model, we have to switch to non-linear (decreasing returns) versions of the technology equations.

\section{Decreasing marginal returns}

The corner solutions of the previous section arise because of the linear nature 
of the combined technology contour. This linear CTC is the result of assumption of the constant returns to scale with respect to the amount of human capital. One additional unit of human capital leads to a proportional change in the technology growth rate values. Jones (1995) criticises the assumption of constant returns with respect to the use of human capital. According to Jones (1995), empirical evidence shows that, 'The assumption embedded in the technology equation that the growth rate of the economy is proportional to the level of resources devoted to $R \& D$ is obviously false.' One of the solutions Jones suggests in order to improve the empirical results of the R\&D-based models is to introduce decreasing returns with respect to human capital. In order to do this, we have reformulated the technology generation equations somewhat,

$$
\begin{aligned}
\frac{d A}{d t} & =\delta_{A}[(z-u) H]^{\gamma_{A}} A \\
\frac{d q}{d t} & =\delta_{q}[u H]^{\gamma_{q}} q
\end{aligned}
$$

where $\gamma_{\mathrm{A}} \leq 1$ and $\gamma_{q} \leq 1$. Note that, whereas Jones (1995) introduces decreasing returns on the level of knowledge, we have decreasing returns solely with respect to human capital. Only in our case the result is a concave CTC. Using Figure 2 we can predict what will happen to the solution of this model at least in the context of the two-step approach. With diminishing returns to scale (DRS) the combined technology contour will be concave (line II), in which case optimization results in one unique solution, point $S$ (see Figure 3), implying that both types of technological change are present.

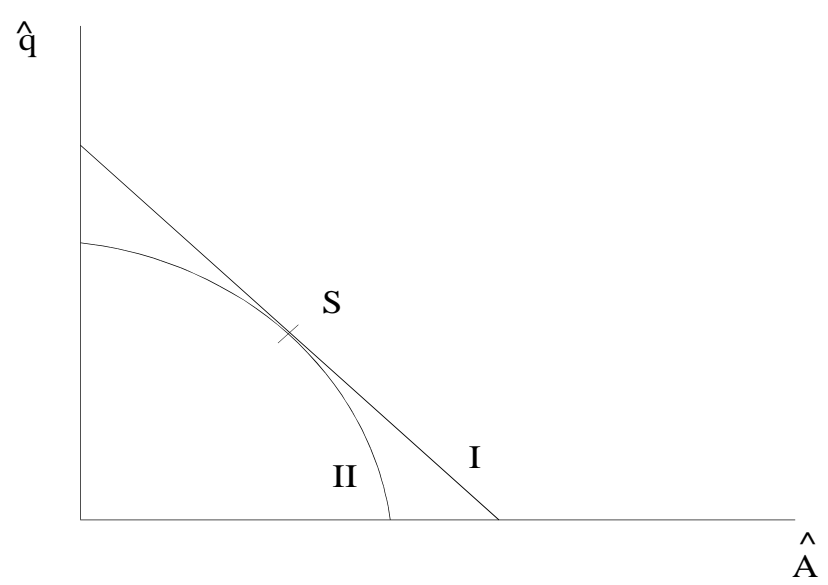

Figure 3 Choice of Technology with DRS

The combined technology contour (CTC) can be derived from the technology equations (5.1) and the iso-TFP-growth-profile (ITP) from equation (4.1). At the optimal combination of technology types, denoted by point $S$ in Figure 3, the slopes of the two curves have to be equal. For a given value of $z$, the 
two-step approach can be used to obtain the efficient values of $\hat{q}$ and $\hat{A}$ (which will only become 'optimal' values by a suitable choice of $z$ ) according to the technology generation equations (5.1). Substituting $\hat{q}(z)$ and $\hat{A}(z)$ into $\hat{T}=\hat{A}+\left(\frac{1-\alpha}{\alpha}\right) \hat{q}$ provides the 'efficient' dynamic constraint,

$$
\frac{d T}{d t}=T\left[\hat{A}(z)+\left(\frac{1-\alpha}{\alpha}\right) \hat{q}(z)\right]
$$

in terms of $z$, which value can now be determined in the usual way ${ }^{10}$. Note that the dynamic constraint on $T$, as given by (5.2), replaces the original dynamic constraints on $A$ and $q$, as given by (5.1).

Unfortunately the model becomes rather difficult to solve analytically with different values for $\gamma_{\mathrm{q}}$ and $\gamma_{\mathrm{A}}$. Hence we will focus first on a special case. In this case, $\gamma_{\mathrm{A}}=1$ and $\gamma_{\mathrm{q}}<1$. This means that the generation of totally new technologies has constant returns to scale with respect to human capital, i.e Romer's case. We expand the Romer model with an equation that describes the quality aspect of technological change. The case in which $\gamma_{\mathrm{A}}<1$ and $\gamma_{\mathrm{q}}=1$ is completely equivalent. However, this would mean that there are diminishing returns in the generation of new technologies, while the quality improvement has constant returns to scale. Since the results of both cases are almost identical we will only focus on the case where $\gamma_{\mathrm{A}}=1$ and $\gamma_{\mathrm{q}}<1^{11}$.

\section{An asymmetric model of combined technological change}

An extra equation describing the increase in the quality of existing technologies is added to the simplified Romer (1990) model we used in section 1. This equation exhibits decreasing returns to scale. From equation (5.2) we know that the exact formulation of the dynamic constraint on $T$ follows from the individual dynamic constraints of $A$ and $q$. The restrictions $\gamma_{\mathrm{A}}=1$ and $\gamma_{\mathrm{q}}<1$ imply that the growth rate of $A$ depends on $z$ whereas the growth rate of $q$ is independent of $z$. This asymmetric allocation of a marginal unit of human capital can be interpreted in terms of the marginal growth productivities (see Figure 4). In case of the generation of better quality, the $\mathrm{MGP}_{q}$, i.e. $\frac{\partial \hat{T}}{\partial(u H)}=\left(\frac{1-\alpha}{\alpha}\right) \frac{\partial \hat{q}}{\partial(u H)}$ falls for increasing values of $(u H)$, whereas

\footnotetext{
${ }^{10}$ A detailed derivation of the results can be found in Appendix E.

${ }^{11}$ Appendix $F$ shows the detailed derivation of the combined model with $\gamma_{A}<1$ and $\gamma_{q}=1$.
} 
the $\operatorname{MGP}_{\mathrm{A}}$, i.e. $\frac{\partial \hat{T}}{\partial[(z-u) H]}=\frac{\partial \hat{A}}{\partial[(z-u) H]}=\delta_{A}$ is constant. Hence, as long as $\frac{\partial \hat{T}}{\partial(u H)}>\frac{\partial \hat{T}}{\partial[(z-u) H]}$ it follows that any increase in human capital should be allocated to $q$ up to the point where $\frac{\partial \hat{T}}{\partial(u H)}=\delta_{A}$, i.e. point $Q$ in Figure 4 . Further increases in $(u H)$ would contribute less to growth than increases in $[(z-u) H]$.

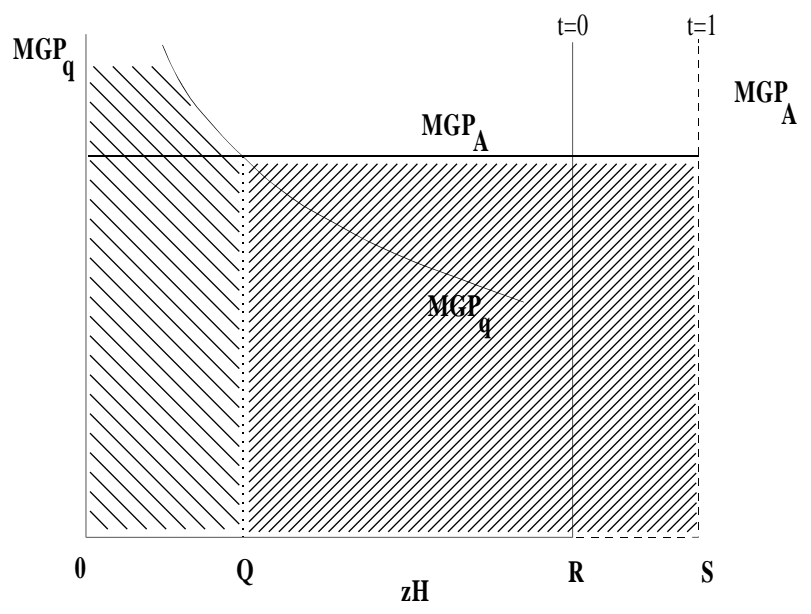

Figure 4 Asymmetrical allocation of a marginal unit of human capital

Hence, $\delta_{\mathrm{A}}$ functions as a kind of lower limit to the marginal growth productivity of human capital. Note that in the Romer (1990) model the marginal growth productivity was equal to this lower limit $\delta_{\mathrm{A}}$. Therefore, we can conclude that the Romer (1990) result can be regarded as the lower limit of our results in case of combined technological change with $\gamma_{A}=1$ and $\gamma_{q}<1$. If the amount of human capital allocated to the generation of technological change, $(z H)$, increases at time $t=1$, from point $R$ to $S$ in Figure 4, it follows immediately that the extra amount of human capital is only allocated to the generation of new technologies, in this case where $\gamma_{\mathrm{A}}=1$ and $\gamma_{\mathrm{q}}<1$.

Thus, an increase in $z$ means an outward shift of the CTC since more human capital is available for technological change. The ITP does not change due to an increase in $z$. A marginal increase in $z$ does not affect the growth rate of $q$, only the growth rate of $A$. This asymmetrical shift of the CTC takes place because of the asymmetry in the technology equations and thus because of the 
assumptions regarding the values of $\gamma_{\mathrm{A}}$ and $\gamma_{\mathrm{q}}{ }^{12}$.

We can now derive the equations for $z, u$ and the various growth rates going through some straight forward calculations which are based on the notion that the ITP, being a function of $z$ and the various productivity parameters and using the assumption of efficient allocation of human capital, essentially defines a dynamic combined technology constraint which can be used to reduce the three-sector model to a 'standard' two-sector framework as used by Romer (1990) and Lucas (1988) (see Appendix E).

The equation for $z$, which is the share of the total stock of human capital that is devoted to technological change, is

$$
z=\frac{(1-\theta)\left[\left(\frac{1-\alpha}{\alpha}\right) \delta_{q} \Psi^{\gamma_{q}}-\delta_{A} \Psi\right]}{\theta H \delta_{A}}+\frac{\delta_{A} H-\rho}{\theta H \delta_{A}}
$$

where $\Psi$ is implicitly defined in terms of the parameters in the model (see Appendix E) and defines the amount of human capital allocated to quality generation. The last part of this equation is equal to the fraction found by Romer (equation (1.7)). Thus, by adding an extra technology equation for the improvement of quality results in a comparable fraction. Recall that $z$ is the fraction of human capital used for combined technological change and is therefore comparable to Romer's fraction $v$. The equivalence between the two shares can be explained in an even better way if we let $\delta_{\mathrm{q}}$ go to zero, i.e. essentially eliminating the technology quality equation. In that case $\Psi$ goes to zero and the share of human capital devoted to the generation of technological change will go to the Romer share - cf. Appendix E where we show that $\Psi=u H$.

The steady state growth rate of this model equals,

$g=\frac{\left(\frac{1-\alpha}{\alpha}\right) \delta_{q} \Psi^{\gamma_{q}}+\delta_{A}(H-\Psi)-\rho}{\theta}$

Using the result $\Psi=u H$, this equation can be easily interpreted if we look at

\footnotetext{
${ }^{12}$ In the case, where $\gamma_{\mathrm{A}}<1$ and $\gamma_{\mathrm{q}}=1$ the same asymmetry will occur for the same reasons. But this time the growth of $A$ is independent of $z$ whereas the growth of $q$ increases with $z$. Only in the case where both R\&D sectors exhibit diminishing returns (to the same extent), the shift of the CTC will be symmetric.
} 
the marginal growth productivities of human capital ${ }^{13}$,

$$
g=\frac{\left[\frac{1}{\gamma_{q}} M G P_{q}-M G P_{A}\right] u H}{\theta}+\frac{\delta_{A} H-\rho}{\theta}
$$

The second term of the RHS of this equation is exactly the Romer steady state growth rate. The first term of the RHS is the effect of adding an extra technology generating equation featuring decreasing returns to the Romer (1990) model. Since in the steady state equilibrium the MGPs have to be equal (see Appendix H), it follows that the first part of the RHS has to be positive. Therefore, we can conclude again that the Romer specification is a special case of our specification, and that the Romer growth rate is a lower limit to the optimal growth rate with combined technological change.

In the next section we solve the model for $\gamma_{\mathrm{q}}=\gamma_{\mathrm{A}}=\gamma$. This results in an equation for $z$ that cannot be solved analytically. However, by investigating the properties of this equation we can nonetheless give some relations between the various variables and parameters. The two cases discussed here give an idea of the way in which we solved the problem and also provide the background for the evaluation of the results found in case $\gamma_{\mathrm{q}}=\gamma_{\mathrm{A}}=\gamma$.

\section{Decreasing returns in both technology equations}

Since the previous case showed the asymmetrical shift of the CTC for an increase in $z$, the most interesting case, from an analytical and theoretical point of view, is the case where both $\gamma_{\mathrm{q}}$ and $\gamma_{\mathrm{A}}$ have identical values and are smaller than one. This is also important in the face of the notion that there is no a priori reason to assume the effectiveness of $R \& D$ efforts to be principally different in both applications. The CTC will shift symmetrically (see Figure 5) in case of an increase in $z$, i.e. the share of human capital devoted to technological change. The results based on $\gamma_{\mathrm{q}}=\gamma_{\mathrm{A}}=\gamma<1$ can be

\footnotetext{
${ }^{13}$ The marginal growth productivity of the amount of human capital allocated to the generation of new technologies is equal to$$
\frac{\partial \hat{Y}}{\partial[(z-u) H]}=\delta_{A}
$$

since $\gamma_{\mathrm{A}}$ was assumed to be equal to one. The marginal growth productivity of the amount of human capital allocated to the improvement of existing technologies is equal to$$
\frac{\partial \hat{Y}}{\partial(u H)}=\gamma_{q}\left(\frac{1-\alpha}{\alpha}\right) \delta_{q}(u H)^{\gamma_{q}-1}
$$ 
found in Appendix G. As can be seen from equations (G2) and (G3) in Appendix $\mathrm{G}$, both the growth rate of $A$ and $q$ depend on the fraction $z$. This is also clear from Figure 5, where an increase in $z$ results in a shift from $S$ to $S^{*}$. This implies an increase in the growth rates of $A$ and $q$. Dynamic optimization results in equation (G15). Unfortunately, this equation in $z$ does not give an explicit solution for $z$, but for given values of the relevant parameters, numerical values of $z$ can readily be obtained.

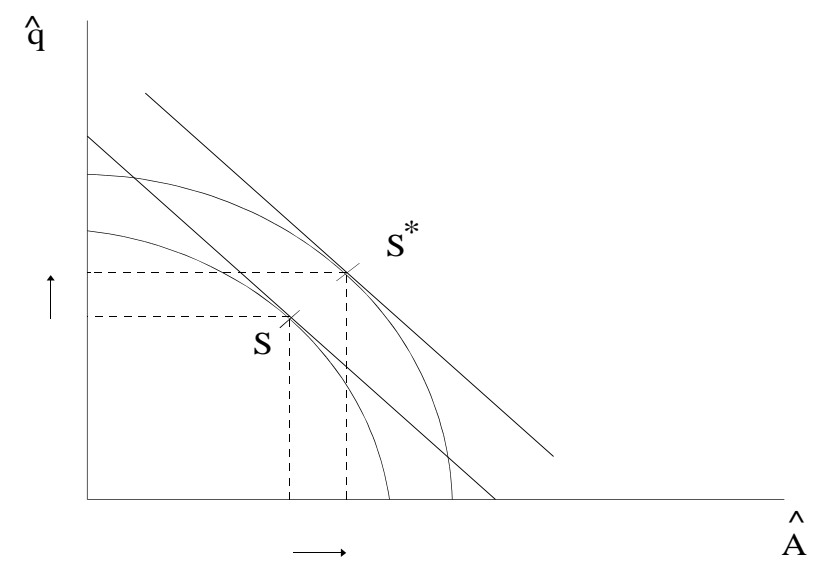

Figure 5 Symmetrical shift of the CTC

But we can also analyze the properties of this equation by means of simple graphs, for equation (G15) can be rewritten as (see also Appendix G) $A+B z=z^{1-\gamma}$

with

$$
A=\frac{\gamma \varepsilon}{\rho} \quad B=\frac{\varepsilon(1-\theta-\gamma)}{\rho}
$$

where $\varepsilon$ can be expressed in the parameters of the model (see Appendix G). The right-hand side (RHS) depends only on $z$ and $\gamma$ while the left-hand side (LHS) depends on $z$ and several other parameters. Since $\varepsilon>0, \gamma>0$ and $\rho>0, A$ is always positive.

The sign of $B$ depends on the value of $\theta$ and $\gamma$. If $\theta+\gamma<1, B$ will be positive and otherwise $B$ will be negative. This means that we have to distinguish between two possible cases with respect to the sign of $B$. In Figure 6 the case in which $A>0$ and $B>0$ is depicted. A positive $B$ implies a positive slope of the LHS. In this case we have three possibilities depending on the value of the intercept $A$. If $A$ is relatively large, no economically relevant solution will be possible. The LHS $^{\mathrm{A}}$ lies above the RHS. If $A$ is relatively small, it is possible to have two solutions $\left(\right.$ LHS $\left.^{\mathrm{C}}\right)$. Only in the 
special case where the slopes of the RHS and the LHS are equal $\left(\mathrm{LHS}^{\mathrm{B}}\right)$ there is one unique value of $z$,

$$
\begin{aligned}
& B=(1-\gamma) z^{-\gamma} \\
& z=\left[\frac{(1-\gamma) \rho}{\varepsilon(1-\theta-\gamma)}\right]^{\frac{1}{\gamma}}
\end{aligned}
$$

Since $z$ has to be positive, it follows from equation (6.2) that $\theta+\gamma<1$.

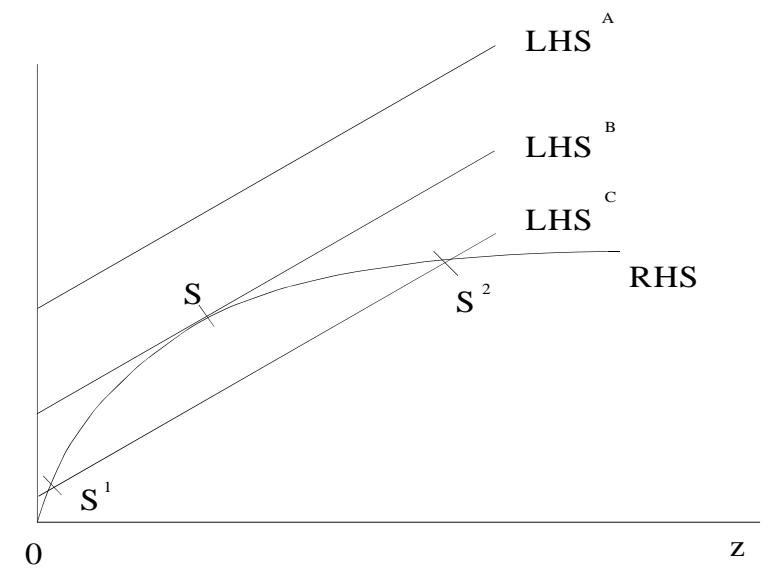

Figure 6 Graphical analysis of share equation $(A>0, B>0)$

Since in this case $\frac{d z}{d \rho}>0$, this solution is not relevant from an economic point of view, for a higher rate of time preference should raise current consumption relative to future consumption, which requires a rise in the fraction of human capital devoted to final production, and therefore a fall in the value of $z$. Although it is not possible to find an exact value for the solutions if the LHS and the RHS do intersect $\left(\mathrm{LHS}^{\mathrm{B}}\right)$, we can nonetheless perform a graphical analysis as in Figure 7.

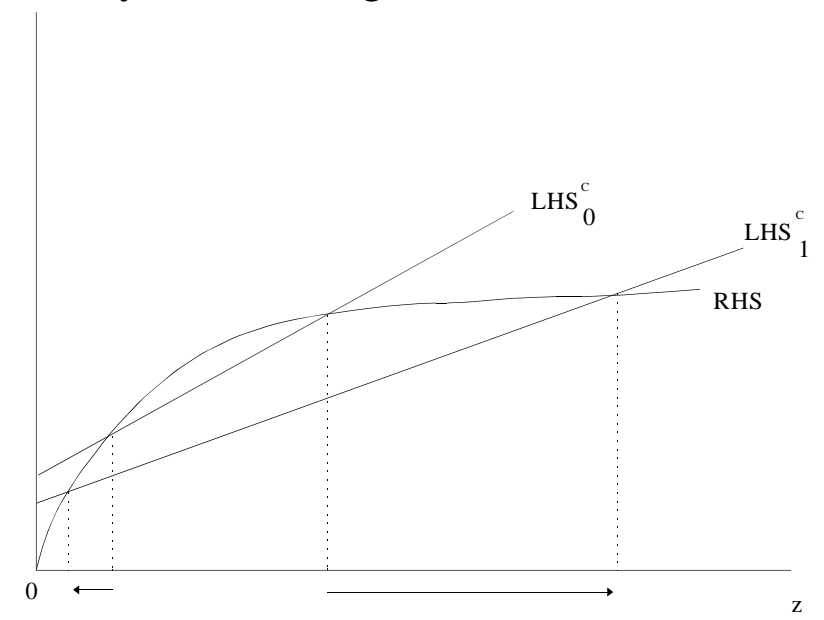

Figure 7 The effect of an increase in $\rho$ on $z$ when $B>0$ 
An increase in $\rho$ implies a decrease in both the intercept $A$ and the slope $B$ of the $\mathrm{LHS}^{\mathrm{B}}$ : $\mathrm{LHS}_{0}{ }^{\mathrm{B}}$ shifts to $\mathrm{LHS}_{1}{ }^{\mathrm{B}}$. As can be seen from Figure 7, the effect of an increase in $\rho$ results in a smaller $z$, as one would expect, in case of the smallest root and in a larger value of $z$ in case of the largest root. This means that only the smallest root shows the desired economically relevant behaviour. Another solution to (6.1) results when $A>0$ and $B<0$. This implies a negative slope for the LHS. Contrary to the previous case, this must result in only one point of intersection. Again we can say more about this solution if we look at the effect of an increase in the rate of time preference. This will cause the LHS to shift downwards while its slope is decreased at the same time.

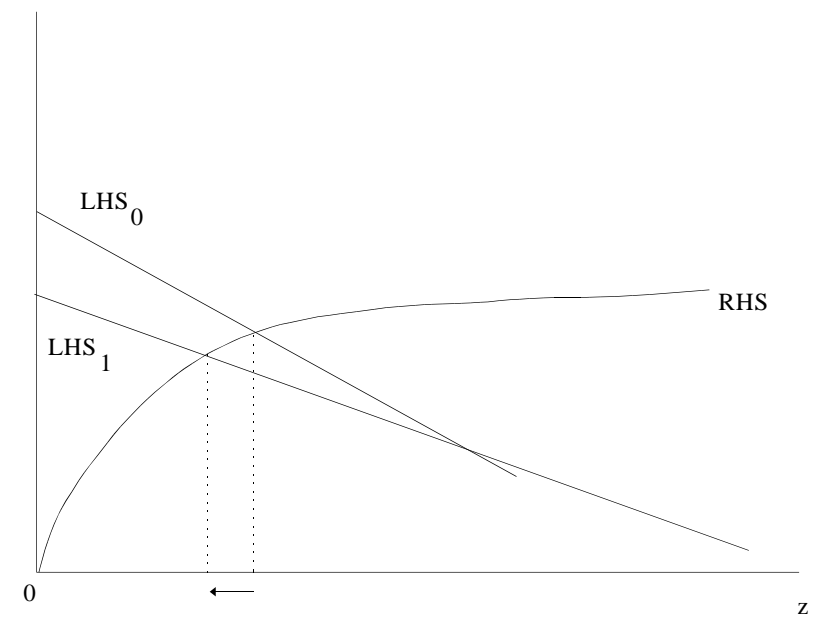

Figure 8 The effect of an increase in $\rho$ on $z$ when $B<0$

As can be seen in Figure 8, a higher value of $\rho$ results in a smaller value of $z$. Thus, we have found a second economically relevant solution ${ }^{14}$.

As before, also in this case the steady state growth rate of this model, $g$ equals the growth rate of $Y, K, C$ and $T$. From equation (G12) in Appendix G, we know that the growth rate of $T$, however, still depends positively on $z$. And so, also in this more generalized framework the growth rate still resembles the previous growth rates we found.

\footnotetext{
${ }^{14}$ Note that it is possible that an increase in $\rho$ leads to a 'rotation' of LHS $_{1}$ in an upward direction such that the downward shift in the intercept of LHS $_{1}$ is more than compensated. This would result in an increase of the equilibrium value of $z$ rather than a decrease. However, one can rule this possibility out on logical grounds, see Appendix $\mathrm{H}$.
} 


\section{Summary and conclusions}

In this paper we have presented an optimal growth model with two types of technological change. The first type generates new technologies, as in the Romer (1990) model, while the second type generates quality improvements, as in the Aghion \& Howitt (1992) model. Solving our model with decreasing returns technology equations results in a combined technology effect. In the steady state both types of technological change are present. The actual amounts of basic research and quality improvement depend on the different marginal growth productivities of human capital of the technologies involved. In section 3 it has been shown that a true technology mix could not be obtained in case of linear technology equations. By defining a two-step approach which provides a better insight in the optimization process and which helps to simplify the solution of the model, we found that even when introducing non-linearities in the technology equations, the model could still be solved. In sections 5 and 6 we described the results of this model. Although we continued to use the two step approach the same results where found applying the usual optimization method with Hamiltonians. However, the two-step approach did not only simplify the calculation of the steady state solutions and share values, but also provided an extra analytical tool. By graphically depicting the combined technology contour (CTC) and the isoTFP-profile (ITP) the results could be predicted and interpreted.

The idea to incorporate both notions of technological change resulted in a model in which the growth rate found by Romer (1990) can be regarded as a lower limit to optimal growth. Thus, by combining the different types of technological change, depending on their marginal growth productivities of human capital, it is possible to generate higher growth rates. The optimal growth rate, however, now depends not only on the optimal allocation of human capital between current production on the one hand and future production on the other, but also on the optimal allocation of human capital between basic research and quality improvements.

Again based on their marginal growth productivities, one could imagine an 'optimal' mix of both types of technological change. This would enable us, at least in the long run, to say something about the desired type of technological change.

The general framework which is presented here can be extended in various ways suggested by international trade theory. The Rybczinsky theorem, for instance, awaits a straight forward technology interpretation. But more importantly, future extensions of this framework should enable us to study the 
more pressing problem of the trade-off between basic and applied research in the face of asymptotic efficiency increases in energy conversion. For, this asymptotic behaviour is implied by the laws of nature, while being ignored by economists for reasons of analytical convenience. 


\section{Appendix A}

\section{Derivation of the simplified Romer model}

Note that the capital aggregate in (1.2) can be seen as a linear homogeneous CES aggregate representing effective capital $K^{e}$, with,

$K^{e}=\left[\sum_{i=1}^{A} x_{i}^{1-\alpha}\right]^{\frac{1}{1-\alpha}}$

In order to get the highest amount of effective capital for a given amount of physical capital, it is necessary to distribute 'raw' capital evenly over all the different blueprints. This follows directly from the concavity and symmetry of $K^{e}$ in $x_{i}$. Hence, it follows that

$$
\bar{x}=x_{i}=\frac{K}{\eta A}
$$

where $1 / \eta$ is the 'productivity' of raw capital ${ }^{15}$. Substituting (A2) in (A1) we find

$K^{e}=\left[\sum_{i=1}^{A}\left(\frac{K}{A}\right)^{1-\alpha}\right]^{\frac{1}{1-\alpha}}=\left[A\left(\frac{K}{A}\right)^{1-\alpha}\right]^{\frac{1}{1-\alpha}}=A^{\frac{\alpha}{1-\alpha}} K$

Hence, the amount of effective capital is proportional to the amount of physical capital. Moreover, it also depends positively on the number of blueprints, where the blueprint elasticity of effective capital is equal to $\frac{\alpha}{1-\alpha}$. This elasticity depends positively on $\alpha$, because a higher value of $\alpha$ increases the 'curvature' of $x_{i}^{1-\alpha}$ for small values of $x_{i}$ and hence it increases the contribution of the concavity of $K^{e}$ in $x_{i}$ to output growth for a given growth rate of the number of blueprints (note that continuous and positive growth in $A$ implies, ceteris paribus, a continuous fall in $x_{i}=\frac{K}{A}$ ). Using (A3), the production function can be written as,

$Y=[(1-v) H]^{\alpha} \cdot K^{e^{1-\alpha}}=[(1-v) H]^{\alpha} \cdot K^{1-\alpha} \cdot A^{\alpha}$

Because Romer (1990) assumes that total capital is cumulative forgone consumption $(C)$ this means that capital grows with,

\footnotetext{
${ }^{15}$ For reasons of simplicity we will assume that $\eta=1$, i.e. it takes one unit of foregone consumption to create one unit of a capital good.
} 
$\frac{d K}{d t}=Y-C$

This aggregation of the individual types of capital goods (designs) to a generalized capital expression, which consists of capital and knowledge, results in a production function that is similar to Lucas (1988). Since they use the same framework, the results found by Romer (1990) and Lucas (1988) can be considered as equivalent.

Using equations (1.1), (1.5) and (1.6) we can construct the following Hamiltonian $\left(\phi^{16}\right)$, in which $v$ and $C$ are the control variables and $K$ and $A$ the state variables,

$\phi=e^{-\rho t} \frac{C^{1-\theta}-1}{1-\theta}+\lambda_{0}\left([(1-v) H]^{\alpha} \cdot K^{1-\alpha} \cdot A^{\alpha}-C\right)+\lambda_{1} \delta v H A$

The first order conditions with respect to the control variables are,

$$
\begin{array}{ll}
\frac{\partial \phi}{\partial C}=e^{-\rho t} \cdot C^{-\theta}-\lambda_{0}=0 \quad \Rightarrow \quad \lambda_{0}=e^{-\rho t} \cdot C^{-\theta} \\
\frac{\partial \phi}{\partial v}=-\lambda_{0} \frac{\alpha Y}{(1-v)}+\lambda_{1} \delta H A=0 \quad \Rightarrow \quad \lambda_{0}=\frac{\lambda_{1} \delta H(1-v) A}{\alpha Y}
\end{array}
$$

The equations of motion for the costate variables are,

$$
\begin{aligned}
& \frac{\partial \phi}{\partial K}=\frac{-d \lambda_{0}}{d t}=\lambda_{0} \frac{(1-\alpha) Y}{K} \Rightarrow-\hat{\lambda}_{0}=\frac{(1-\alpha) Y}{K} \\
& \frac{\partial \phi}{\partial A}=\frac{-d \lambda_{1}}{d t}=\lambda_{0} \frac{\alpha Y}{A}+\lambda_{1} \delta v H \quad \Rightarrow \quad-\hat{\lambda}_{1}=\frac{\lambda_{0}}{\lambda_{1}} \frac{\alpha Y}{A}+\delta v H
\end{aligned}
$$

We can rewrite equation (A10) by substituting the result of equation (A8),

$$
-\hat{\lambda}_{1}=\frac{\delta H(1-v) A}{\alpha Y} \frac{\alpha Y}{A}+\delta v H \quad \Rightarrow \quad-\hat{\lambda}_{1}=\delta H
$$

The steady state is a situation in which the various quantities grow at constant rates. This means in this case that the growth rates of $A, K, C$, and $Y$ have to be constant. The steady state growth rate of human capital is assumed to be zero. (If this would not be the case, then growth would increase with a positive growth of $\mathrm{H}$ ) Rewriting equation (A7) in growth rates,

\footnotetext{
${ }^{16}$ The Hamiltonian is denoted by $\phi$ in order to avoid possible confusion with the $H$ of human capital.
} 
$\hat{\lambda}_{0}=-\rho-\theta \hat{C}$

Rewriting equation (A8) in growth rates,

$\hat{\lambda}_{0}=\hat{\lambda}_{1}+\hat{A}-\hat{Y}$

Rewriting the production function in growth rates,

$\hat{Y}=(1-\alpha) \hat{K}+\alpha \hat{A}$

From (A11) we know that the growth rate of $\lambda_{1}$ is constant. Since the growth rates of $A$ and $Y$ are also constant in the steady state we can conclude that $\lambda_{0}$ is constant. Applying these steady state conditions we get from equation (A9),

$$
\hat{\hat{\lambda}}_{0}=\hat{Y}-\hat{K}=0 \Rightarrow \hat{Y}=\hat{K}
$$

Since the growth rate of $K$ equals the growth rate of $Y$, it follows that the growth rate of $A$ equals the growth rate of $Y$. Using equation (A3),

$$
\frac{C}{Y}=\frac{Y}{Y}-\hat{K} \frac{K}{Y}
$$

and because $K / Y$ is constant, we can conclude that $C / Y$ is constant too, and hence the growth rate of $C$ equals the growth rate of $Y$. The common steady state growth rate for this model, $g$, is therefore equal to the growth rates of $A$, $K, Y$ and $C$. This means that equation (A13) can be reduced to,

$$
\hat{\lambda}_{0}=\hat{\lambda}_{1}
$$

Substituting all the results above in equation (A12), we get

$$
\begin{aligned}
& \hat{\lambda}_{1}=-\rho-\theta \hat{A} \quad \Rightarrow \quad-\delta H=-\rho-\theta \delta v H \quad \Rightarrow \\
& v=\frac{\delta H-\rho}{\theta \delta H} \quad \Rightarrow \quad \hat{A}=\frac{\delta H-\rho}{\theta}
\end{aligned}
$$




\section{Appendix B}

Derivation of the technology model with quality improvements

Again we will derive the necessary conditions for a social optimum which maximizes consumer utility $(U)$ in equation (1.4). To solve this optimization problem we construct the following Hamiltonian, in which $v$ and $C$ are the control variables and $K$ and $q$ the state variables,

$$
\phi=e^{-\rho t} \frac{C^{1-\theta}-1}{1-\theta}+\lambda_{0}\left([(1-v) H]^{\alpha} \cdot(q K)^{1-\alpha} \cdot A^{\alpha}-C\right)+\lambda_{1} \delta v H q
$$

The first order conditions with respect to the control variables are,

$$
\begin{array}{ll}
\frac{\partial \phi}{\partial C}=e^{-\rho t} \cdot C^{-\theta}-\lambda_{0}=0 \quad & \Rightarrow \quad \lambda_{0}=e^{-\rho t} \cdot C^{-\theta} \\
\frac{\partial \phi}{\partial v}=-\lambda_{0} \frac{\alpha Y}{(1-v)}+\lambda_{1} \delta H q=0 & \Rightarrow \quad \lambda_{0}=\frac{\lambda_{1} \delta H(1-v) q}{\alpha Y}
\end{array}
$$

The equations of motion for the costate variables are,

$$
\begin{aligned}
& \frac{\partial \phi}{\partial K}=\frac{-d \lambda_{0}}{d t}=\lambda_{0} \frac{(1-\alpha) Y}{K} \Rightarrow-\hat{\lambda}_{0}=\frac{(1-\alpha) Y}{K} \\
& \frac{\partial \phi}{\partial q}=\frac{-d \lambda_{1}}{d t}=\lambda_{0} \frac{(1-\alpha) Y}{q}+\lambda_{1} \delta v H \quad \Rightarrow \quad-\hat{\lambda}_{1}=\frac{\lambda_{0}}{\lambda_{1}} \frac{(1-\alpha) Y}{q}+\delta v H
\end{aligned}
$$

We can rewrite equation (B5) by substituting the result of equation (B3),

$$
-\hat{\lambda}_{1}=\frac{\delta H(1-v) q}{\alpha Y} \frac{(1-\alpha) Y}{q}+\delta v H \Rightarrow-\hat{\lambda}_{1}=\delta H(1-v) \frac{1-\alpha}{\alpha}+\delta v H
$$

Applying the usual steady state conditions, we find that $\hat{Y}=\hat{K}=\hat{C}$ (see also Appendix A). If we rewrite the production function (2.2) in growth rates and use the fact that in the steady state $\hat{Y}=\hat{K}$, we find the following relation between the growth rate of $Y$ and the growth rate of $q$

$$
\hat{Y}=\frac{1-\alpha}{\alpha} \hat{q}
$$

Note that the relation between the growth rate of $Y$ and the growth rate of $q$ differs from the relation between $\hat{Y}$ and $\hat{A}$ in the previous section. The growth rate of final production is no longer equal to the growth rate of the technological change, but adjusted by a factor $(1-\alpha) / \alpha$. This means that if we 
write equation (B3) in growth rates and replace the growth rate of $Y$ by the adjusted growth rate of $q$, the result is

$$
\hat{\lambda}_{0}=\hat{\lambda}_{1}+\hat{q}^{-} \frac{1-\alpha}{\alpha} \hat{q}
$$

All we have to do now is to rewrite equation (B2) in growth rates and replace $\hat{\lambda}_{0}$ with equation (B8) and $\hat{C}$ by equation (B7). The results in an expression in $\hat{q}$ and $\hat{\lambda}_{1}$, which can be substituted into equations (2.3) and (B6),

$$
\hat{\lambda}_{1}+\hat{q}-\frac{1-\alpha}{\alpha} \hat{q}=-\rho-\theta \frac{1-\alpha}{\alpha} \hat{q}
$$

Rearranging terms, we can calculate the fraction $v$,

$$
\begin{aligned}
& -\delta H \frac{1-\alpha}{\alpha}=-\rho-\theta \frac{1-\alpha}{\alpha} \delta v H \\
& v=\frac{\delta H \frac{1-\alpha}{\alpha}-\rho}{\theta \delta H \frac{1-\alpha}{\alpha}}
\end{aligned}
$$




\section{Appendix C}

A naive model of combined technological change

The production function and technology generation equations, which are part of the intertemporal utility maximization problem are denoted by,

$$
\begin{aligned}
& Y=[(1-u-v) H]^{\alpha} \cdot(q K)^{1-\alpha} \cdot A^{\alpha} \\
& \frac{d A}{d t}=\delta_{A} v H A \\
& \frac{d q}{d t}=\delta_{q} u H q
\end{aligned}
$$

Note that it is now necessary to divide total human capital between final production (fraction is $1-u-v$ ), the generation of new technologies (fraction $v$ ) and the improvement of already existing technologies (fraction $u$ ). Also note, that the Cobb-Douglas production function enables us to collect the impact of the various forms of technological change on output into one measure by defining a combined technology variable,

$$
T=A q^{\frac{1-\alpha}{\alpha}} \Leftrightarrow \hat{T}=\hat{A}+\left(\frac{1-\alpha}{\alpha}\right) \hat{q}
$$

Again we will derive the necessary conditions for a social optimum which maximizes consumer utility $(U)$. To solve this optimization problem we construct the following Hamiltonian, in which $v, u$ and $C$ are the control variables and $K, A$ and $q$ the state variables,

$$
\begin{gathered}
\phi=e^{-\rho t} \frac{C^{1-\theta}-1}{1-\theta}+\lambda_{0}\left([(1-u-v) H]^{\alpha} \cdot(q K)^{1-\alpha} \cdot A^{\alpha}-C\right)+ \\
\lambda_{1} \delta_{A} v H A+\lambda_{2} \delta_{q} u H q
\end{gathered}
$$

The first order conditions with respect to the control variables are,

$$
\begin{array}{ll}
\frac{\partial \phi}{\partial C}=e^{-\rho t} \cdot C^{-\theta}-\lambda_{0}=0 \quad \Rightarrow \quad \lambda_{0}=e^{-\rho t} \cdot C^{-\theta} \\
\frac{\partial \phi}{\partial v}=-\lambda_{0} \frac{\alpha Y}{(1-u-v)}+\lambda_{1} \delta_{A} H A=0 \quad \Rightarrow \quad \lambda_{0}=\frac{\lambda_{1} \delta_{A} H(1-u-v) A}{\alpha Y} \\
\frac{\partial \phi}{\partial u}=-\lambda_{0} \frac{\alpha Y}{(1-u-v)}+\lambda_{2} \delta_{q} H q=0 \quad \Rightarrow \quad \lambda_{0}=\frac{\lambda_{2} \delta_{q} H(1-u-v) q}{\alpha Y}
\end{array}
$$

The equations of motion for the costate variables are, 


$$
\begin{aligned}
& \frac{\partial \phi}{\partial K}=\frac{-d \lambda_{0}}{d t}=\lambda_{0} \frac{(1-\alpha) Y}{K} \Rightarrow-\hat{\lambda}_{0}=\frac{(1-\alpha) Y}{K} \\
& \frac{\partial \phi}{\partial A}=\frac{-d \lambda_{1}}{d t}=\lambda_{0} \frac{\alpha Y}{A}+\lambda_{1} \delta_{A} v H \Rightarrow-\hat{\lambda}_{1}=\frac{\lambda_{0}}{\lambda_{1}} \frac{\alpha Y}{A}+\delta_{A} v H \\
& \frac{\partial \phi}{\partial q}=\frac{-d \lambda_{2}}{d t}=\lambda_{0} \frac{(1-\alpha) Y}{q}+\lambda_{2} \delta_{q} u H \quad \Rightarrow \quad-\hat{\lambda}_{2}=\frac{\lambda_{0}}{\lambda_{2}} \frac{(1-\alpha) Y}{q}+\delta_{q} u H
\end{aligned}
$$

We can rewrite equation (C8) by substituting the result of equation (C5),

$$
-\hat{\lambda}_{1}=\frac{\delta_{A} H(1-u-v) A}{\alpha Y} \frac{\alpha Y}{A}+\delta_{A} v H \quad \Rightarrow \quad-\hat{\lambda}_{1}=\delta_{A} H(1-u)
$$

We can rewrite equation (C9) by substituting the result of equation (C6),

$$
\begin{aligned}
& -\hat{\lambda}_{2}=\frac{\delta_{q} H(1-u-v) q}{\alpha Y} \frac{(1-\alpha) Y}{q}+\delta_{q} u H \quad \Rightarrow \\
& -\hat{\lambda}_{2}=\delta_{q} H \frac{1-\alpha}{\alpha}(1-u-v)+\delta_{q} u H
\end{aligned}
$$

Applying the steady state conditions we get from equations (C7), (C4), (C5) and (C6),

$$
\begin{aligned}
& -\hat{\lambda}_{0}=\hat{Y}-\hat{K}=0 \Rightarrow \hat{Y}=\hat{K} \\
& \hat{\lambda}_{0}=-\rho-\theta \hat{C} \\
& \hat{\lambda}_{0}=\hat{\lambda}_{1}+\hat{A}-\hat{Y} \\
& \hat{\lambda}_{0}=\hat{\lambda}_{2}+\hat{q}-\hat{Y}
\end{aligned}
$$

From equations (C14) and (C15) we know that,

$$
\hat{\lambda}_{1}-\hat{\lambda}_{2}=\hat{q}-\hat{A}
$$

Substituting the previous results in this equation we get

$$
\begin{aligned}
& -(1-u) \delta_{A} H+\frac{1-\alpha}{\alpha}(1-u-v) \delta_{q} H+u \delta_{q} H=u \delta_{q} H-v \delta_{A} H \quad \Leftrightarrow \\
& (1-u-v)\left(\frac{1-\alpha}{\alpha} \delta_{q}-\delta_{A}\right)=0
\end{aligned}
$$

This equation can only hold in two cases. Either the fractions $v$ and $u$ add up 
to one, but this implies that final production will become zero since no human capital is left to be used in final production, or in the second case, which is the relevant one from an economic point of view, the marginal growth productivities of human capital have to be equal. However, in the case of technology generation equations which are linear in human capital this is the exception rather than the rule. Moreover, when $\left(\frac{1-\alpha}{\alpha}\right) \delta_{q}-\delta_{A}=0$, both applications of human capital would generate the same (and constant) MGP, in which case $u$ and $v$ are not uniquely defined. 


\section{Appendix D}

Formal proof of the equivalence between the two-step approach and the standard approach

Rewrite the production function using,

$$
\begin{aligned}
& q^{\prime}=\ln (q) \quad \Rightarrow \quad \frac{d q^{\prime}}{d t}=\hat{q} \\
& A^{\prime}=\ln (A) \quad \Rightarrow \quad \frac{d A^{\prime}}{d t}=\hat{A}
\end{aligned}
$$

to,

$$
Y=[(1-z) H]^{\alpha} e^{(1-\alpha) q^{\prime}+\alpha A^{\prime}} K^{1-\alpha}
$$

This transformation enables us to derive optimal control results directly in terms of $\hat{q}$ and $\hat{A}$, i.e. the concepts used in the context of the two-step approach.

The associated technology generation equations and the Hamiltonian are,

$$
\begin{aligned}
& \frac{d A^{\prime}}{d t} \triangleq \hat{A}=f[(z-u) H] \quad f^{\prime}>0, f^{\prime \prime} \leq 0 \\
& \frac{d q^{\prime}}{d t} \triangleq \hat{q}=g[u H] \quad g^{\prime}>0, g^{\prime \prime} \leq 0 \\
& \Phi=e^{-\rho t} \frac{C^{1-\theta}-1}{1-\theta}+\lambda_{0}(Y-C)+\lambda_{1} \hat{q}+\lambda_{2} \hat{A}
\end{aligned}
$$

The first order conditions with respect to the control variables are,

$$
\begin{aligned}
& \frac{\partial \Phi}{\partial C}=0 \quad \Rightarrow \quad \hat{\lambda}_{0}=-\rho-\theta \hat{C} \\
& \frac{\partial \Phi}{\partial z}=-\lambda_{0} \frac{\alpha Y}{(1-z)}+\lambda_{1} \frac{\partial \hat{A}}{\partial z}=0 \\
& \frac{\partial \Phi}{\partial u}=\lambda_{1} \frac{\partial \hat{q}}{\partial u}+\lambda_{2} \frac{\partial \hat{A}}{\partial(z-u)} \frac{\partial(z-u)}{\partial u}=0
\end{aligned}
$$

The equations of motion for the costate variables are, 


$$
\begin{aligned}
& \frac{\partial \Phi}{\partial q^{\prime}}=-\frac{d \lambda_{1}}{d t}=\lambda_{0}(1-\alpha) Y \\
& \frac{\partial \Phi}{\partial A^{\prime}}=-\frac{d \lambda_{2}}{d t}=\lambda_{0} \alpha Y
\end{aligned}
$$

(D7) and (D8) follow from the fact that $\hat{q}$ and $\hat{A}$ are independent of $q^{\prime}$ and $A^{\prime}$, respectively.

In the steady state, $z$ and $u$ are constant. Hence,

$$
\frac{\partial \hat{A}}{\partial(z-u)}=f^{\prime} \quad \text { and } \quad \frac{\partial \hat{q}}{\partial u}=g^{\prime}
$$

are constant too. Differentiating (D6) with respect to time we find,

$$
\frac{d \lambda_{1}}{d t} \frac{\partial \hat{q}}{\partial u}-\frac{d \lambda_{2}}{d t} \frac{\partial \hat{A}}{\partial(z-u)}=0
$$

The slope of the CTC implied by $\hat{q}$ and $\hat{A}$ as given by (D3) is by definition equal to,

$$
\frac{d \hat{q}}{d \hat{A}}=-\frac{\frac{\partial \hat{q}}{\partial u}}{\frac{\partial \hat{A}}{\partial(z-u)}}=-\frac{\frac{d \lambda_{2}}{d t}}{\frac{d \lambda_{1}}{d t}}
$$

by virtue of (D10). Substitution of (D7) and (D8) into (D11) results in,

$$
\frac{d \hat{q}}{d \hat{A}}=-\frac{\alpha}{1-\alpha}
$$

Hence the optimality conditions of the two-step approach are contained in the first order conditions of the Hamiltonian problem and the steady state assumption. Note that (D12) and (D11) taken together imply that,

$$
\left(\frac{1-\alpha}{\alpha}\right) \frac{\partial \hat{q}}{\partial u}=\frac{\partial \hat{A}}{\partial(z-u)}
$$

i.e. in the optimum situation the marginal growth productivities of human capital should be the same in both technology applications of human capital. 


\section{Appendix E}

A two-step model of combined technological change with $\gamma_{q}<1$ and $\gamma_{A}=1$

The slopes of the CTC and the ITP have to be equal in the optimal point $S$,

$$
-\frac{1}{\gamma_{q}} \frac{\delta_{A}}{\delta_{q}}\left(\frac{\hat{q}}{\delta_{q}}\right)^{\frac{1}{\gamma_{q}}-1}=-\left(\frac{1-\alpha}{\alpha}\right)
$$

Using (E1), we can derive an equation for $\hat{q}$

$$
\frac{\hat{q}}{\delta_{q}}=\left[\left(\frac{\gamma_{q} \delta_{q}(1-\alpha)}{\delta_{A} \alpha}\right)^{\frac{1}{1-\gamma_{q}}}\right]^{\gamma_{q}} \triangleq \Psi^{\gamma_{q}}
$$

where $\Psi$ is implicitly defined in terms of the parameters of the model and is equal to the amount of human capital allocated to quality generation. We can now derive an equation for $\hat{A}$ by substituting the result of (E2) in the CTC, giving

$$
\hat{A}=\delta_{A}\left(z H-\left(\frac{\hat{q}}{\delta_{q}}\right)^{\frac{1}{\gamma_{q}}}\right)
$$

This results in

$$
\hat{A}=\delta_{A}(z H-\Psi)
$$

Substituting both equations for $\hat{q}$ and $\hat{A}$ into the ITP results in

$$
\hat{T}=\left(\frac{1-\alpha}{\alpha}\right) \delta_{q} \Psi^{\gamma_{q}}-\delta_{A} \Psi+\delta_{A} z H
$$

This is the ITP we will use in the optimization problem. The Hamiltonian associated with this problem is

$$
\begin{aligned}
\Phi=e^{-\rho t} \frac{C^{1-\theta}-1}{1-\theta}+ & \lambda_{0}\left([(1-z) T H]^{\alpha} K^{1-\alpha}-C\right)+ \\
& \lambda_{1} T\left[\left(\frac{1-\alpha}{\alpha}\right) \delta_{q} \Psi^{\gamma_{q}}+\delta_{A}(z H-\Psi)\right]
\end{aligned}
$$


The first order conditions with respect to the control variables are

$$
\begin{aligned}
& \frac{\partial \Phi}{\partial C}=e^{-\rho t} C^{-\theta}-\lambda_{0}=0 \quad \Rightarrow \quad \lambda_{0}=e^{-\rho t} C^{-\theta} \\
& \frac{\partial \Phi}{\partial z}=-\lambda_{0} \frac{\alpha Y}{1-z}+\lambda_{1} T H \delta_{A}=0 \quad \Rightarrow \quad \lambda_{0}=\frac{(1-z) \lambda_{1} T H \delta_{A}}{\alpha Y}
\end{aligned}
$$

The equations of motion for the costate variables are

$$
\begin{aligned}
& \frac{\partial \Phi}{\partial K}=\frac{-d \lambda_{0}}{d t}=\lambda_{0} \frac{(1-\alpha) Y}{K} \Rightarrow-\hat{\lambda}_{0}=\frac{(1-\alpha) Y}{K} \\
& \frac{\partial \Phi}{\partial T}=\frac{-d \lambda_{1}}{d t}=\lambda_{0} \frac{\alpha Y}{T}+\lambda_{1}\left[\left(\frac{1-\alpha}{\alpha}\right) \delta_{q} \Psi^{\gamma_{q}}+\delta_{A}(z H-\Psi)\right]
\end{aligned}
$$

Substituting (E8) in (E10) gives

$$
\begin{aligned}
& \frac{-d \lambda_{1}}{d t}=\frac{(1-z) \lambda_{1} T H \delta_{A}}{\alpha Y} \frac{\alpha Y}{T}+\lambda_{1}\left[\left(\frac{1-\alpha}{\alpha}\right) \delta_{q} \Psi^{\gamma_{q}}+\delta_{A}(z H-\Psi)\right] \\
& -\hat{\lambda}_{1}=(1-z) H \delta_{A}+\left(\frac{1-\alpha}{\alpha}\right) \delta_{q} \Psi^{\gamma_{q}}+\delta_{A}(z H-\Psi)
\end{aligned}
$$

In the steady state, the growth rates of $Y, K, C$ and $T$ are the same. This means that we can rewrite equation (E7) in

$$
\begin{aligned}
& \hat{\lambda}_{0}=-\rho-\theta \hat{C} \\
& \hat{\lambda}_{1}=-\rho-\theta \hat{T}
\end{aligned}
$$

By substituting (E11) into (E12) we get an equation for $\hat{T}$

$$
\hat{T}=\frac{\left(\frac{1-\alpha}{\alpha}\right) \delta_{q} \Psi^{\gamma_{q}}+\delta_{A}(H-\Psi)-\rho}{\theta}
$$

We now have two equations for $\hat{T}$, (E5) and (E13), which can be used to solve for $z$. Given $z$, we can then solve for $u$ and the various growth rates.

$$
\left(\frac{1-\alpha}{\alpha}\right) \delta_{q} \Psi^{\gamma_{q}}+\delta_{A}(z H-\Psi)=\frac{\left(\frac{1-\alpha}{\alpha}\right) \delta_{q} \Psi^{\gamma_{q}}+\delta_{A}(H-\Psi)-\rho}{\theta}
$$




$$
\begin{aligned}
& z=\frac{\left(\frac{1-\alpha}{\alpha}\right) \delta_{q} \Psi^{\gamma_{q}}(1-\theta)+\delta_{A} \Psi(\theta-1)+\delta_{A} H-\rho}{\theta H \delta_{A}} \\
& z=\frac{(1-\theta)\left[\left(\frac{1-\alpha}{\alpha}\right) \delta_{q} \Psi^{\gamma_{q}}-\delta_{A} \Psi\right]+\delta_{A} H-\rho}{\theta H \delta_{A}}
\end{aligned}
$$

Substituting this result for $z$ into equation (E4), we get the growth rate of $A$

$$
\hat{A}=\frac{(1-\theta)\left(\frac{1-\alpha}{\alpha}\right) \delta_{q} \Psi^{\gamma_{q}}+\delta_{A}(H-\Psi)-\rho}{\theta}
$$

Since

$$
u=z-\frac{\hat{A}}{\delta_{A}}
$$

we can now calculate $u$ by substituting equations (E14) and (E16). The result is

$$
u=\frac{\Psi}{H}
$$

The final fraction we have to calculate is $z-u$

$$
z-u=\frac{(1-\theta)\left(\frac{1-\alpha}{\alpha}\right) \delta_{q} \Psi^{\gamma_{q}}+\delta_{A}(H-\Psi)-\rho}{\theta H \delta_{A}}
$$

The steady state growth rate is equal to

$$
g=\frac{\left(\frac{1-\alpha}{\alpha}\right) \delta_{q} \Psi^{\gamma_{q}}+\delta_{A}(H-\Psi)-\rho}{\theta}
$$




\section{Appendix F}

A two-step model of combined technological change with $\gamma_{A}<1$ and $\gamma_{q}=1$

The slopes of the CTC and the ITP have to be equal in the optimal point $\mathrm{S}$, given that $\gamma_{\mathrm{q}}=1$ and $\gamma_{\mathrm{A}}<1$, hence

$$
-\gamma_{a} \frac{\delta_{A}}{\delta_{q}}\left(z-\frac{\hat{q}}{\delta_{q}}\right)^{\gamma_{A}-1}=-\left(\frac{1-\alpha}{\alpha}\right)
$$

Using (F1), we can derive an equation for $\hat{q}$

$$
\begin{aligned}
& z H-\frac{\hat{q}}{\delta_{q}}=\left(\frac{\delta_{q}(1-\alpha)}{\gamma_{A} \delta_{A} \alpha}\right)^{\frac{1}{\gamma_{A}-1}} \equiv \Psi \\
& \hat{q}=\delta_{q}(z H-\Psi)
\end{aligned}
$$

Note that $\Psi$ is again defined in terms of the parameters of the model. However, $\Psi$ now represents the amount of human capital allocated to the generation of new technologies. We can now derive an equation for $\hat{A}$ by substituting the result of (F2) in the CTC denoted by equation (4.4),

$$
\hat{A}=\delta_{A} \Psi^{\gamma_{A}}
$$

Substituting both equations for $\hat{q}$ and $\hat{A}$ into the ITP results in,

$$
\hat{T}=\delta_{A} \Psi^{\gamma_{A}}-\left(\frac{1-\alpha}{\alpha}\right) \delta_{q} \Psi+\left(\frac{1-\alpha}{\alpha}\right) \delta_{q} z H
$$

This is the ITP we will use in the optimization problem. The Hamiltonian associated with this problem is,

$$
\begin{aligned}
\Phi=e^{-\rho t} \frac{C^{1-\theta}-1}{1-\theta}+ & \lambda_{0}\left([(1-z) T H]^{\alpha} K^{1-\alpha}-C\right)+ \\
& \left.\lambda_{1} T\left[\left(\frac{1-\alpha}{\alpha}\right) \delta_{q}(z H-\Psi)+\delta_{A} \Psi^{\gamma_{A}}\right)\right]
\end{aligned}
$$

The first order conditions with respect to the control variables are

$$
\frac{\partial \Phi}{\partial C}=e^{-\rho t} C^{-\theta}-\lambda_{0}=0 \quad \Rightarrow \quad \lambda_{0}=e^{-\rho t} C^{-\theta}
$$




$$
\frac{\partial \Phi}{\partial z}=-\lambda_{0} \frac{\alpha Y}{1-z}+\lambda_{1} T H\left(\frac{1-\alpha}{\alpha}\right) \delta_{q}=0 \Rightarrow \lambda_{0}=\frac{(1-z) \lambda_{1} T H\left(\frac{1-\alpha}{\alpha}\right) \delta_{q}}{\alpha Y}
$$

The equations of motion for the costate variables are,

$$
\begin{aligned}
& \frac{\partial \Phi}{\partial K}=\frac{-d \lambda_{0}}{d t}=\lambda_{0} \frac{(1-\alpha) Y}{K} \Rightarrow-\hat{\lambda}_{0}=\frac{(1-\alpha) Y}{K} \\
& \frac{\partial \Phi}{\partial T}=\frac{-d \lambda_{1}}{d t}=\lambda_{0} \frac{\alpha Y}{T}+\lambda_{1}\left[\left(\frac{1-\alpha}{\alpha}\right) \delta_{q}(z H-\Psi)+\delta_{A} \Psi^{\gamma_{A}}\right]
\end{aligned}
$$

Substituting (F7) in (F9) gives,

$$
\begin{aligned}
& \left.\frac{-d \lambda_{1}}{d t}=\frac{(1-z) \lambda_{1} T H\left(\frac{1-\alpha}{\alpha}\right) \delta_{q}}{\alpha Y} \frac{\alpha Y}{T}+\lambda_{1}\left[\left(\frac{1-\alpha}{\alpha}\right) \delta_{q}(z H-\Psi)+\delta_{A} \Psi^{\gamma_{A}}\right)\right] \\
& -\hat{\lambda}_{1}=\left(\frac{1-\alpha}{\alpha}\right) \delta_{q}(H-\Psi)+\delta_{A} \Psi^{\gamma_{A}}
\end{aligned}
$$

In the steady state, the growth rates of $Y, K, C$ and $T$ are the same and $z$ is a constant. This means that we can rewrite equation (F6) in

$$
\begin{aligned}
& \hat{\lambda}_{0}=-\rho-\theta \hat{C} \\
& \hat{\lambda}_{1}=-\rho-\theta \hat{T}
\end{aligned}
$$

By substituting (F10) into (F11), we get a equation for $\hat{T}$

$$
\hat{T}=\frac{\left(\frac{1-\alpha}{\alpha}\right) \delta_{q}(H-\Psi)+\delta_{A} \Psi^{\gamma_{A}}-\rho}{\theta}
$$

We now have two equations for $\hat{T}$, (F4) and (F12), which can be used to solve $z$. From this we can solve for $u$ and the various growth rates.

$$
\left(\frac{1-\alpha}{\alpha}\right) \delta_{q}(z H-\Psi)+\delta_{A} \Psi^{\gamma_{A}}=\frac{\left(\frac{1-\alpha}{\alpha}\right) \delta_{q}(H-\Psi)+\delta_{A} \Psi^{\gamma_{A}}-\rho}{\theta} \Rightarrow
$$




$$
\begin{gathered}
z=\frac{\left(\frac{1-\alpha}{\alpha}\right) \delta_{q}(H-\Psi+\theta \Psi)+\delta_{A} \Psi^{\gamma_{A}}(1-\theta)-\rho}{\theta H\left(\frac{1-\alpha}{\alpha}\right) \delta_{q}} \Rightarrow \\
z=\frac{(1-\theta)\left[\delta_{A} \Psi^{\gamma_{A}}-\left(\frac{1-\alpha}{\alpha}\right) \delta_{q} \Psi\right]}{\theta H\left(\frac{1-\alpha}{\alpha}\right) \delta_{q}}+\frac{\delta_{q}\left(\frac{1-\alpha}{\alpha}\right) H-\rho}{\theta H\left(\frac{1-\alpha}{\alpha}\right) \delta_{q}}
\end{gathered}
$$

Substituting this result for $z$ into equation (F2), we get the growth rate of $q$

$$
\hat{q}=\frac{(1-\theta) \delta_{A} \Psi^{\gamma_{A}}-\left(\frac{1-\alpha}{\alpha}\right) \delta_{q} \Psi}{\theta\left(\frac{1-\alpha}{\alpha}\right)}+\frac{\delta_{q}\left(\frac{1-\alpha}{\alpha}\right) H-\rho}{\theta\left(\frac{1-\alpha}{\alpha}\right)}
$$

From (F14) and the equation of motion of $q$, it follows that,

$$
u=\frac{\hat{q}}{\delta_{q}} H=\frac{(1-\theta) \delta_{A} \Psi \gamma_{A}-\left(\frac{1-\alpha}{\alpha}\right) \delta_{q} \Psi}{\theta\left(\frac{1-\alpha}{\alpha}\right) \delta_{q} H}+\frac{\left(\frac{1-\alpha}{\alpha}\right) \delta_{q} H-\rho}{\theta\left(\frac{1-\alpha}{\alpha}\right) \delta_{q} H}
$$

The final fraction we have to calculate is $z-u$

$$
z-u=H \Psi \equiv H\left(\frac{\delta_{q}(1-\alpha)}{\gamma_{A} \delta_{A} \alpha}\right)^{\frac{1}{\gamma_{A}-1}}
$$

The steady state growth rate is equal to,

$$
g=\frac{\delta_{A} \Psi^{\gamma_{A}}+\left(\frac{1-\alpha}{\alpha}\right) \delta_{q}(H-\Psi)-\rho}{\theta}
$$




\section{Appendix G}

A two-step model of combined technological change with $\gamma_{A}=\gamma_{q}=\gamma<1$

The slopes of the CTC and the ITP have to be the same in the optimal point $S$, given that $\gamma_{q}=\gamma_{A}=\gamma<1$

$$
-\gamma \frac{\delta_{A}}{\delta_{q}}\left(z-\left(\frac{\hat{q}}{\delta_{q}}\right)^{\frac{1}{\gamma}}\right)^{\gamma-1} \cdot \frac{1}{\gamma}\left(\frac{\hat{q}}{\delta_{q}}\right)^{\frac{1}{\gamma}-1}=-\left(\frac{1-\alpha}{\alpha}\right)
$$

From this we can derive an equation for $\hat{q}^{17}$,

$z^{-\gamma} \frac{\hat{q}}{\delta_{q}}=\left[\left(\frac{\delta_{q}}{\delta_{A}}\left(\frac{1-\alpha}{\alpha}\right)\right)^{\frac{1}{\gamma-1}}+1\right]^{-\gamma} \equiv \chi \quad \Rightarrow \quad \hat{q}=\delta_{q} \chi z^{\gamma}$

Note that $\chi$ is defined in terms of the parameters of the model. We can now derive an equation for $\hat{A}$ by substituting the result of (G2) in the CTC given by equation (4.4)

$$
\hat{A}=\delta_{A} z^{\gamma}\left(1-\chi^{\frac{1}{\gamma}}\right)^{\gamma}
$$

Substituting both equations for $\hat{q}$ and $\hat{A}$ into the ITP results in

$$
\hat{T}=z^{\gamma}\left(\delta_{A}\left(1-\chi^{\frac{1}{\gamma}}\right)^{\gamma}+\left(\frac{1-\alpha}{\alpha}\right) \delta_{q} \chi\right)
$$

This is the ITP used in the optimization problem. The Hamiltonian is,

$$
\begin{aligned}
\Phi=e^{-\rho t} \frac{C^{1-\theta}-1}{1-\theta}+ & \lambda_{0}\left([(1-z) T]^{\alpha} K^{1-\alpha}-C\right)+ \\
& \lambda_{1} T z^{\gamma}\left(\delta_{A}\left(1-\chi^{\frac{1}{\gamma}}\right)^{\gamma}+\left(\frac{1-\alpha}{\alpha}\right) \delta_{q} \chi\right)
\end{aligned}
$$

The first order conditions with respect to the control variables are,

\footnotetext{
${ }^{17}$ We have normalized human capital to a value of one. This simplifies the analysis without changing the basic outcome.
} 


$$
\begin{aligned}
& \frac{\partial \Phi}{\partial C}=e^{-\rho t} C^{-\theta}-\lambda_{0}=0 \Rightarrow \lambda_{0}=e^{-\rho t} C^{-\theta} \\
& \frac{\partial \Phi}{\partial z}=-\lambda_{0} \frac{\alpha Y}{1-z}+\lambda_{1} T \gamma z^{\gamma-1}\left(\delta_{A}\left(1-\chi^{\frac{1}{\gamma}}\right)^{\gamma}+\left(\frac{1-\alpha}{\alpha}\right) \delta_{q} \chi\right)=0 \Rightarrow \\
& \lambda_{0}=\frac{(1-z) \lambda_{1} T \gamma z^{\gamma-1}\left(\delta_{A}\left(1-\chi^{\frac{1}{\gamma}}\right)^{\gamma}+\left(\frac{1-\alpha}{\alpha}\right) \delta_{q} \chi\right)}{\alpha Y}
\end{aligned}
$$

The equations of motion for the costate variables are,

$$
\begin{aligned}
& \frac{\partial \Phi}{\partial K}=\frac{-d \lambda_{0}}{d t}=\lambda_{0} \frac{(1-\alpha) Y}{K} \Rightarrow-\hat{\lambda}_{0}=\lambda_{0} \frac{(1-\alpha) Y}{K} \\
& \frac{\partial \Phi}{\partial T}=\frac{-d \lambda_{1}}{d t}=\lambda_{0} \frac{\alpha Y}{T}+\lambda_{1} z^{\gamma}\left(\delta_{A}\left(1-\chi^{\frac{1}{\gamma}}\right)^{\gamma}+\left(\frac{1-\alpha}{\alpha}\right) \delta_{q} \chi\right)
\end{aligned}
$$

Substituting (G7) in (G9) gives,

$$
-\hat{\lambda}_{1}=\left[\gamma z^{\gamma-1}+z^{\gamma}(1-\gamma)\right]\left[\delta_{A}\left(1-\chi^{\frac{1}{\gamma}}\right)^{\gamma}+\left(\frac{1-\alpha}{\alpha}\right) \delta_{q} \chi\right]
$$

In the steady state, the growth rates of $Y, K, C$ and $T$ are the same and $z$ is a constant. This means that we can rewrite equation (G6) in

$$
\begin{aligned}
& \hat{\lambda}_{0}=-\rho-\theta \hat{C} \\
& \hat{\lambda}_{1}=-\rho-\theta \hat{T}
\end{aligned}
$$

By simple and straight forward substitution we get an equation for $\hat{T}$

$$
\hat{T}=\frac{\left[\gamma z^{\gamma-1}+z^{\gamma}(1-\gamma)\right]\left[\delta_{A}\left(1-\chi^{\frac{1}{\gamma}}\right)^{\gamma}+\left(\frac{1-\alpha}{\alpha}\right) \delta_{q} \chi\right]-\rho}{\theta}
$$

We can now substitute equation (G4). To simplify we set,

$$
\varepsilon=\delta_{A}\left(1-\chi^{\frac{1}{\gamma}}\right)^{\gamma}+\left(\frac{1-\alpha}{\alpha}\right) \delta_{q} \chi
$$

This results in the following equation, 
$z^{\gamma} \varepsilon=\frac{\left[\gamma z^{\gamma-1}+z^{\gamma}(1-\gamma)\right] \varepsilon-\rho}{\theta}$

$z^{\gamma}-\frac{\gamma}{\theta-1+\gamma} z^{\gamma-1}=\frac{-\rho}{\varepsilon(\theta-1+\gamma)}$

or

$z^{1-\gamma}=\frac{z-\frac{\gamma}{\theta-1+\gamma}}{\frac{-\rho}{\varepsilon(\theta-1+\gamma)}}=\frac{\gamma \varepsilon}{\rho}+\frac{(1-\theta-\gamma-1) \varepsilon}{\rho} z$ 


\section{Appendix $\mathbf{H}$}

Parameter analyses

Note that in order for $\frac{d z}{d \rho}$ to be negative, it is necessary that at the 'old' value of $z$, i.e. $z^{*}$, but at the 'new' (increased) value of $\rho$ the $\operatorname{LHS}\left(z^{*}, \rho\right)$, should be smaller than the $\operatorname{RHS}\left(z^{*}, \rho\right)$. Hence, we would require

$$
\frac{d A}{d \rho}+\frac{d B}{d \rho} z^{*}<0 \Rightarrow z^{*}<\frac{\gamma}{\Theta+\gamma-1}
$$

Since $\theta \leq 1$ by assumption $z^{*}<\frac{\gamma}{\theta+\gamma-1}$ cannot in any way be a binding constraint for $z^{*}$, i.e. for any logically feasible value of $z^{*} \leq 1$, the requirement that $z^{*}<\frac{\gamma}{\theta+\gamma-1}$ is automatically satisfied. Therefore, if $z^{*}$ is a feasible solution, then it exhibits the relevant economic behaviour. The corollary of this result is that if a solution $z^{*}$ is feasible, then

$$
\frac{d A}{d \rho}+\frac{d B}{d \rho} z^{*}>0 \Rightarrow z^{*}>\frac{\gamma}{\Theta+\gamma-1}>1
$$

which would make $z^{*}$ infeasible, contrary to our assumption. Therefore we can rule out the possibility that $\frac{d z}{d \rho}>0$. Similar conclusions hold with respect to the other parameters. 


\section{References}

-Aghion, P., Howitt, P., (1992), A model of growth through creative destruction, Econometrica, 60, 2, pp.323-351.

-Barro, R.J., Sala-i-Martin, X., (1995), Economic growth, McGraw-Hill, New York.

-Grossman, G.M., Helpman, E., (1991), Innovation and growth in the global economy, Cambridge MA, MIT Press.

-Helpman, E., Trajtenberg, M. (1994), A Time to Sow and a Time to Reap: Growth Based on General Purpose Technologies, CIAR Program in Economic Growth and Policy, Working Paper No. 32.

-Jones, C.I., (1995), R\&D based models of economic growth, Journal of Political Economy, 103, 4, pp. 759-784.

-Kennedy, C., (1964), Induced bias in innovation and the theory of distribution, Economic Journal, pp. 541-7.

-Lucas, R.E., (1988), On the mechanics of economic development, Journal of Monetary Economics, 22, pp. 3-42.

-Romer, P.M., (1990), Endogenous technological change, Journal of Political Economy, 98, 5, part II, S71-S102.

-Solow, R.M., (1956), A contribution to the theory of economic growth, Quarterly Journal of Economics, 70, 1, pp. 65-94.

-Verberne, P.H., (1995), Economic Models Concerning the Effects of Reducing $\mathrm{CO}_{2}$ Emissions: A Survey, in: Diederen, P., Kemp, R., Verberne, P., Ziesemer, T. and Zon, A. van, Energy Technologies, Environmental Policy and Competitiveness, Final report for JOULE II programme of the European Commission, DG XII. 